\title{
Implicator-Conjunctor Based Models of Fuzzy Rough Sets: Definitions and Properties
}

\author{
Lynn D'eer ${ }^{1}$, Nele Verbiest ${ }^{1}$, Chris Cornelis ${ }^{1,2}$, and Lluis Godo ${ }^{3}$ \\ 1 Department of Applied Mathematics, Computer Science and Statistics, Ghent \\ University, Krijgslaan 281 (S9), B-9000 Gent, Belgium \\ \{Lynn.Deer, Nele.Verbiest\}@UGent . be \\ 2 Department of Computer Science and Artificial Intelligence, University of Granada, \\ Calle del Periodista Daniel Saucedo Aranda s/n, 18071 Granada, Spain \\ chriscornelis@ugr.es \\ 3 IIIA - CSIC, Campus de la UAB s/n, 08193 Bellaterra, Spain \\ godo@iia.csic.es
}

\begin{abstract}
Ever since the first hybrid fuzzy rough set model was proposed in the early 1990's, many researchers have focused on the definition of the lower and upper approximation of a fuzzy set by means of a fuzzy relation. In this paper, we review those proposals which generalize the logical connectives and quantifiers present in the rough set approximations by means of corresponding fuzzy logic operations. We introduce a general model which encapsulates all of these proposals, evaluate it w.r.t. a number of desirable properties, and refine the existing axiomatic approach to characterize lower and upper approximation operators.

Keywords: fuzzy sets, rough sets, hybridization, lower and upper approximation, implication, conjunction, axiomatic approach
\end{abstract}

\section{Introduction}

Fuzzy sets [1] generalize classical or crisp sets in a sense that objects can be assigned intermediary membership degrees to a set or relation, drawn from a partially ordered set, typically $[0,1]$. On the other hand, rough sets $[2]$ characterize a set of objects by means of a lower and an upper approximation, taking into account an equivalence relation that represents indiscernibility between objects. Both theories have fostered broad research communities and have been applied in a wide range of settings. It was recognized early on that they are complementary, rather than competitive; a first hybrid fuzzy rough set model was proposed by Dubois and Prade [3] in 1990. Now, more than 20 years later, interest in fuzzy rough sets is thriving; this is mainly thanks to their proven application potential in machine learning, and in particular in feature selection [4-6] and instance selection [7].

Fuzzy-rough hybridization has been pursued in a variety of ways; in this paper, we focus on the most common approach, i.e., using fuzzy logical extensions of the Boolean implication and conjunction, along with infimum and supremum as extensions of the universal and existential quantifiers. This idea sparked the 
seminal proposal in [3], and since then many papers [8-18] have focused on the refinement of this model using both constructive approaches, which propose new definitions of approximation operators, and axiomatic approaches, which set forth a set of axioms or desirable properties, and characterize the operators that satisfy them.

It was found that through a deliberate choice of fuzzy logical operators, and the use of a similarity relation (also called fuzzy equivalence relation) to model approximate indiscernibility, most properties of the original rough set model can be maintained $[8,9]$; on the other hand, from a practical point of view, the use of similarity relations is not always convenient (see e.g. [6]), and as De Cock et al. [16] argued, they cause part of the hybridization potential to remain unexplored. Moreover, also in the crisp case, various types of binary relations have been considered to replace the indiscernibility equivalence relation, see e.g. $[19,20]$. For all of these reasons, several other authors considered fuzzy rough set models based on general fuzzy relations $[10-15,17]$.

In this paper, we unify all these approaches under the umbrella of a general implicator-conjunctor based fuzzy rough set model, imposing minimal restrictions on the approximations. After recalling some preliminaries in Section 2, we present the definitions of the approximations in Section 3, and give a chronological overview of special cases studied in the literature. In Section 4, we evaluate the model w.r.t. desirable properties, while in Section 5, we refine the axiomatic approach of $\mathrm{Wu}$ et al. [13], weakening some of its conditions and proposing an alternative characterization that caters specifically to residual implications. Finally, in Section 6, we conclude and outline future work.

\section{Preliminaries}

\subsection{Fuzzy Logical Connectives}

A conjunctor is a mapping $\mathcal{C}:[0,1]^{2} \rightarrow[0,1]$ which is increasing in both arguments and which satisfies $\mathcal{C}(0,0)=\mathcal{C}(0,1)=\mathcal{C}(1,0)=0$ and $\mathcal{C}(1,1)=1$. It is called a border conjunctor if it satisfies $\mathcal{C}(1, x)=x$ for all $x$ in $[0,1]$. A commutative, associative border conjunctor $\mathcal{T}$ is called a $t$-norm.

A disjunctor is a mapping $\mathcal{D}:[0,1]^{2} \rightarrow[0,1]$ which is increasing in both arguments and which satisfies $\mathcal{D}(1,0)=\mathcal{D}(0,1)=\mathcal{D}(1,1)=1$ and $\mathcal{D}(0,0)=0$. It is called a border disjunctor if it satisfies $\mathcal{D}(0, x)=x$ for all $x$ in $[0,1]$. A commutative, associative border disjunctor $\mathcal{S}$ is called a $t$-conorm.

A negator is a decreasing mapping $\mathcal{N}:[0,1] \rightarrow[0,1]$ which satisfies $\mathcal{N}(0)=1$ and $\mathcal{N}(1)=0$. It is involutive if for all $x \in[0,1], \mathcal{N}(\mathcal{N}(x))=x$. The standard negator $\mathcal{N}_{s}$ is defined by, for $x$ in $[0,1], \mathcal{N}_{s}(x)=1-x$.

Given an involutive negator $\mathcal{N}$, a conjunctor $\mathcal{C}$ and a disjunctor $\mathcal{D}$, the $\mathcal{N}$ dual of $\mathcal{C}$ is a disjunctor $\mathcal{D}_{\mathcal{C}, \mathcal{N}}$, defined by $\mathcal{D}_{\mathcal{C}, \mathcal{N}}(x, y)=\mathcal{N}(\mathcal{C}(\mathcal{N}(x), \mathcal{N}(y)))$, and the $\mathcal{N}$-dual of $\mathcal{D}$ is a conjunctor $\mathcal{C}_{\mathcal{D}, \mathcal{N}}$, defined by $\mathcal{C}_{\mathcal{D}, \mathcal{N}}(x, y)=\mathcal{N}(\mathcal{D}(\mathcal{N}(x)$, $\mathcal{N}(y))$ ), for all $x, y$ in $[0,1]$. It can be verified that the $\mathcal{N}$-dual of a t-norm is a t-conorm, and vice versa. 
An implicator $\mathcal{I}$ is a mapping $\mathcal{I}:[0,1]^{2} \rightarrow[0,1]$ satisfying $\mathcal{I}(1,0)=0$, $\mathcal{I}(1,1)=\mathcal{I}(0,1)=\mathcal{I}(0,0)=1$ which is decreasing in the first and increasing in the second argument. If $\mathcal{I}$ satisfies $\mathcal{I}(1, x)=x$ for all $x$ in $[0,1]$, it is called a border implicator, and if it satisfies the exchange principle, $\mathcal{I}(x, \mathcal{I}(y, z))=I(y, \mathcal{I}(x, z))$ for all $x, y, z$ in $[0,1]$, it is called an EP implicator.

Let $\mathcal{C}, \mathcal{D}$ and $\mathcal{N}$ be a border conjunctor, a disjunctor and a negator respectively. The $S$-implicator $\mathcal{I}_{\mathcal{D}, \mathcal{N}}$ based on $\mathcal{D}$ and $\mathcal{N}$ is defined by, for $x, y$ in $[0,1]$, $\mathcal{I}_{\mathcal{D}, \mathcal{N}}(x, y)=\mathcal{D}(\mathcal{N}(x), y)$. The R-implicator $\mathcal{I}_{\mathcal{C}}$ based on $\mathcal{C}$ is defined by, for $x, y$ in $[0,1], \mathcal{I}_{\mathcal{C}}(x, y)=\sup \{\gamma \in[0,1] \mid \mathcal{C}(x, \gamma) \leq y\}$. Both S-implicators and R-implicators are particular cases of border implicators.

Given an involutive negator $\mathcal{N}$ and an implicator $\mathcal{I}$, the induced conjunctor of $\mathcal{I}$ and $\mathcal{N}$ is a conjunctor $\mathcal{C}_{\mathcal{I}, \mathcal{N}}$ defined by, for $x, y \in[0,1], \mathcal{C}_{\mathcal{I}, \mathcal{N}}(x, y)=$ $\mathcal{N}(\mathcal{I}(x, \mathcal{N}(y))$. It is not necessarily a t-norm.

\subsection{Fuzzy Sets and Relations}

A fuzzy set $A$ in a non-empty universe set $U$ is a mapping $A: U \rightarrow[0,1]$. The collection of all fuzzy sets in $U$ is denoted by $\mathcal{F}(U)$.

Given $\alpha$ in $[0,1]$, the constant (fuzzy) set $\hat{\alpha}$ is defined by, for $x$ in $U, \hat{\alpha}(x)=\alpha$. In the crisp case, the only constant sets are $\emptyset$ and $U$.

Let $A, B \in \mathcal{F}(U)$ and $x \in U$. Given a negator $\mathcal{N}$, the $\mathcal{N}$-complement of $A$ is defined by $\left(\cos _{\mathcal{N}}(A)\right)(x)=\mathcal{N}(A(x))$. Given a conjunctor $\mathcal{C}$ and a disjunctor $\mathcal{D}$, the $\mathcal{C}$-intersection and $\mathcal{D}$-union of $A$ and $B$ are defined by $\left(A \cap_{\mathcal{C}} B\right)(x)=$ $\mathcal{C}(A(x), B(x))$ and $\left(A \cup_{\mathcal{D}} B\right)(x)=\mathcal{D}(A(x), B(x))$. If $\mathcal{C}=\min$ and $\mathcal{D}=\max$, we simply write $\cap$ and $\cup$. Given an implicator $\mathcal{I}$, the $\mathcal{I}$-implication of $A$ and $B$ is defined by $\left(A \Rightarrow_{\mathcal{I}} B\right)(x)=\mathcal{I}(A(x), B(x))$.

A binary fuzzy relation $R$ in $U$ is a fuzzy set in $U \times U$. We define its inverse fuzzy relation $R^{\prime}$ by $R^{\prime}(x, y)=R(y, x)$ for $x, y$ in $U . R$ is called reflexive if $R(x, x)=1$, symmetric if $R(x, y)=R(y, x)$ and inverse serial if $\sup _{x \in U} R(x, y)=1$ for all $y$ in $U$. For a symmetric binary fuzzy relation $R$, it obviously holds that $R=R^{\prime}$.

Given a t-norm $\mathcal{T}, R$ is called $\mathcal{T}$-transitive if for all $x, y$ and $z$ in $U$, $\mathcal{T}(R(x, y), R(y, z)) \leq R(x, z)$. If $R$ is reflexive, symmetric and $\mathcal{T}$-transitive, it is called a $\mathcal{T}$-similarity relation. When $\mathcal{T}=$ min, we shortly speak about a similarity relation. Because the minimum operator is the largest t-norm, a similarity relation is a $\mathcal{T}$-similarity relation for every t-norm $\mathcal{T}$.

\subsection{Lower and Upper Approximations in Rough Set Theory}

A classical or Pawlak approximation space is a couple $(U, R)$ consisting of a nonempty set $U$ and an equivalence relation $R$ in $U$. The rough approximation of a crisp set $A$ in $U$ by $R$ is the pair of sets $(R \downarrow A, R \uparrow A)$ defined by, for $x \in U$,

$$
\begin{aligned}
& x \in R \downarrow A \Leftrightarrow(\forall y \in U)((y, x) \in R \Rightarrow y \in A) \\
& x \in R \uparrow A \Leftrightarrow(\exists y \in U)((y, x) \in R \wedge y \in A) .
\end{aligned}
$$


A pair $\left(A_{1}, A_{2}\right)$ of sets in $U$ is called a rough set in $(U, R)$ if there is a set $A$ in $U$ such that $A_{1}=R \downarrow A$ and $A_{2}=R \uparrow A$. Some of the most important properties of lower and upper approximation in a Pawlak approximation space are listed in the left hand side of Table 2. Note that we denote the complement of a crisp set $A$ by $A^{c}$.

\section{Implicator-Conjunctor Based Model}

Many definitions of fuzzy rough sets emerge by faithfully extending Eqs. (1) and $(2)$ to the $[0,1]$-valued case. In particular, Dubois and Prade worked with a similarity relation $R$, and replaced the Boolean implication and conjunction by the S-implicator $\mathcal{I}_{\max , \mathcal{N}_{s}}$ (Kleene-Dienes implicator) and the minimum tnorm, respectively. In this section, we consider a fuzzy approximation space, i.e., a couple $(U, R)$ consisting of a non-empty set $U$ and a binary fuzzy relation $R$ in $U$, and define a general format for the approximations using implicators and conjunctors.

Definition 1. Let $(U, R)$ be a fuzzy approximation space, $A$ a fuzzy set in $U, \mathcal{I}$ an implicator and $\mathcal{C}$ a conjunctor. The $(\mathcal{I}, \mathcal{C})$-fuzzy rough approximation of $A$ by $R$ is the pair of fuzzy sets $\left(R \downarrow_{\mathcal{I}} A, R \uparrow_{\mathcal{C}} A\right)$ defined by, for $x \in U$,

$$
\begin{aligned}
& \left(R \downarrow_{\mathcal{I}} A\right)(x)=\inf _{y \in U} \mathcal{I}(R(y, x), A(y)) \\
& \left(R \uparrow_{\mathcal{C}} A\right)(x)=\sup _{y \in U} \mathcal{C}(R(y, x), A(y)) .
\end{aligned}
$$

A pair $\left(A_{1}, A_{2}\right)$ of fuzzy sets in $U$ is called a fuzzy rough set in $(U, R)$ if there is a fuzzy set $A$ in $U$ such that $A_{1}=R \downarrow_{\mathcal{I}} A$ and $A_{2}=R \uparrow_{\mathcal{C}} A$.

In Table 1 we give a chronological overview of special cases of the general model. Some authors $[8,15,18]$ actually require lower semicontintuity of $\mathcal{T}$ instead of left-continuity, but by a result from [21] these two notions are equivalent for t-norms. Also, some papers $[10,11,13,17]$ consider fuzzy relations from $U$ to $W$, with both $U$ and $W$ non-empty, finite universes, but here we restrict ourselves to the case $U=W$. As can be seen, Wu et al. [10] were the first to consider general binary fuzzy relations, while Mi and Zhang [11] initiated the use of conjunctors that are not necessarily t-norms. Also note that the t-norm $\mathcal{T}_{\text {cos }}$ used in [18] is defined, for $x, y$ in $[0,1]$, by $\mathcal{T}_{\cos }(x, y)=\max \left(x y-\sqrt{\left(1-x^{2}\right)\left(1-y^{2}\right)}, 0\right)$. Its use is inspired by the fact that some commonly used kernel functions in machine learning are in fact $\mathcal{T}_{\mathrm{cos}^{-} \text {-similarity relations. }}$

\section{Properties}

In the following, we assume that $(U, R),\left(U, R_{1}\right)$ and $\left(U, R_{2}\right)$ are fuzzy approximation spaces, $A$ and $B$ are fuzzy sets in $U, \mathcal{I}$ is an implicator, $\mathcal{C}$ a conjunctor and $\mathcal{N}$ an involutive negator. In the right hand side of Table 2 , we show the 
Table 1. Overview of special cases of the general fuzzy rough set model

\begin{tabular}{|c|c|c|c|}
\hline Model & Conjunctor & Implicator & Relation \\
\hline [3] Dubois \& Prade, 1990 & $\min$ & $\mathcal{I}_{\max , \mathcal{N}_{s}}$ & similarity \\
\hline [8] Morsi \& Yakout, 1998 & left-cont. t-norm $\mathcal{T}$ & $\mathcal{I}_{\mathcal{T}}$ & $\mathcal{T}$-similarity \\
\hline [9] Radzikowska \& Kerre, 2002 & t-norm $\mathcal{T}$ & border implicator $\mathcal{I}$ & similarity \\
\hline [10] Wu et al., 2003 & $\min$ & $\mathcal{I}_{\max , \mathcal{N}_{s}}$ & general \\
\hline [11] Mi \& Zhang, 2004 & $\begin{array}{l}\mathcal{C}_{\mathcal{I}_{\mathcal{T}}, \mathcal{N}_{s}} ; \\
\text { left-cont. t-norm } \mathcal{T}\end{array}$ & $\mathcal{I}_{\mathcal{T}}$ & general \\
\hline [13] $\mathrm{Wu}$ et al., 2005 & cont. t-norm $\mathcal{T}$ & implicator $\mathcal{I}$ & general \\
\hline [14] Pei, 2005 & $\min$ & $\mathcal{I}_{\max , \mathcal{N}_{s}}$ & general \\
\hline [15] Yeung et al., 2005 & left-cont. t-norm $\mathcal{T}$ & $\mathcal{I}_{\mathcal{S}_{T, \mathcal{N}}, \mathcal{N}}, \mathcal{N}$ involutive & general \\
\hline [15] Yeung et al., 2005 & $\begin{array}{l}\mathcal{C}_{\mathcal{I}_{\mathcal{T}}, \mathcal{N}_{s}} ; \\
\text { left-cont. t-norm } \mathcal{T}\end{array}$ & $\mathcal{I}_{\mathcal{T}}$ & general \\
\hline [16] De Cock et al., 2007 & t-norm $\mathcal{T}$ & border implicator $\mathcal{I}$ & general \\
\hline [17] Mi et al., 2008 & cont. t-norm $\mathcal{T}$ & $\mathcal{I}_{\mathcal{S}_{\mathcal{T}, \mathcal{N}_{S}}, \mathcal{N}_{S}}$ & general \\
\hline [18] Hu et al., 2010 & $\begin{array}{l}\text { left-cont. t-norm } \mathcal{T} \\
\text { eft-cont. t-norm } \mathcal{T}\end{array}$ & $\mathcal{I}_{\mathcal{S}_{\mathcal{T}, \mathcal{N}_{s}}, \mathcal{N}_{s}}$ & $\begin{array}{l}\mathcal{T}_{\text {cos }^{-}} \\
\text {similarity }\end{array}$ \\
\hline [18] Hu et al., 2010 & $\begin{array}{l}\mathcal{C}_{\mathcal{I}_{\mathcal{T}}, \mathcal{N}_{s}} ; \\
\text { left-cont. t-norm } \mathcal{T}\end{array}$ & $\mathcal{I}_{\mathcal{T}}$ & $\begin{array}{l}\mathcal{T}_{\text {cos }^{-}} \\
\text {similarity }\end{array}$ \\
\hline
\end{tabular}

extensions of the classical rough set properties to a fuzzy approximation space. We can prove the following propositions, which mainly generalize known results obtained in a restricted setting, see e.g. [9].

Proposition 1. If $\mathcal{C}$ is the induced conjunctor of $\mathcal{I}$ and $\mathcal{N}$, i.e., $\mathcal{C}=\mathcal{C}_{\mathcal{I}, \mathcal{N}}$, then the duality property holds.

Corollary 1. Let $\mathcal{D}$ be the $\mathcal{N}$-dual disjunctor of $\mathcal{C}$. If the pair $(\mathcal{I}, \mathcal{C})$ consists of the $S$-implicator $\mathcal{I}_{\mathcal{D}, \mathcal{N}}$ and the conjunctor $\mathcal{C}$, then the duality property holds.

Corollary 2. Let $\mathcal{T}$ be a left-continuous t-norm and $\mathcal{N}=\mathcal{N}_{\mathcal{I}_{\mathcal{T}}}$. If the pair $(\mathcal{I}, \mathcal{C})$ consists of the $R$-implicator $\mathcal{I}_{\mathcal{T}}$ and the $t$-norm $\mathcal{T}$, then the duality property holds.

To see this corollary, note that $\mathcal{C}_{\mathcal{I}_{\mathcal{T}}, \mathcal{N}}=\mathcal{T}$ indeed holds: for $x, y$ in $[0,1]$, $\mathcal{C}_{\mathcal{I}_{\mathcal{T}}, \mathcal{N}}(x, y)=\mathcal{N}\left(\mathcal{I}_{\mathcal{T}}(x, \mathcal{N}(y))\right)=\mathcal{N}\left(\mathcal{I}_{\mathcal{T}}\left(x, \mathcal{I}_{\mathcal{T}}(y, 0)\right)\right)=\mathcal{N}\left(\mathcal{I}_{\mathcal{T}}(\mathcal{T}(x, y), 0)\right)=$ $\mathcal{N}(\mathcal{N}(\mathcal{T}(x, y)))=\mathcal{T}(x, y)$

Proposition 2. If the pair $(\mathcal{I}, \mathcal{C})$ consists of the $R$-implicator $\mathcal{I}_{\mathcal{T}}$ and the leftcontinuous t-norm $\mathcal{T}$, then the adjointness property holds.

Note that in generalizing the adjointness condition to a fuzzy approximation space, we have replaced $R$ in the right hand side of the equivalence by its inverse fuzzy relation $R^{\prime}$. Clearly, if $R$ is symmetric (which is the case for a Pawlak approximation space), this modification is redundant.

Proposition 3. If $R$ is reflexive, $\mathcal{I}$ is a border implicator and $\mathcal{C}$ is a border conjunctor, then the inclusion property holds. 
Table 2. Properties in a Pawlak approximation space and their corresponding extensions to a fuzzy approximation space.

\begin{tabular}{|c|c|c|}
\hline Name & Pawlak approximation space & Fuzzy approximation space \\
\hline Duality & $\begin{array}{l}R \downarrow A=\left(R \uparrow A^{\mathrm{c}}\right)^{\mathrm{c}} \\
R \uparrow A=\left(R \downarrow A^{\mathrm{c}}\right)^{\mathrm{c}}\end{array}$ & $\begin{array}{l}R \downarrow_{\mathcal{I}} A=\operatorname{co} \mathcal{N}\left(R \uparrow_{\mathcal{C}}(\cos (A))\right) \\
R \uparrow_{\mathcal{C}} A=\cos _{\mathcal{N}}\left(R \downarrow_{\mathcal{I}}(\operatorname{co} \mathcal{N}(A))\right)\end{array}$ \\
\hline Adjointness & $R \uparrow A \subseteq B \Leftrightarrow A \subseteq R \downarrow B$ & $R \uparrow_{\mathcal{C}} A \subseteq B \Leftrightarrow A \subseteq R^{\prime} \downarrow_{\mathcal{I}} B$ \\
\hline Inclusion & $\begin{array}{l}R \downarrow A \subseteq A \\
A \subseteq R \uparrow A\end{array}$ & $\begin{array}{l}R \downarrow_{\mathcal{I}} A \subseteq A \\
A \subseteq R \uparrow_{\mathcal{C}} A\end{array}$ \\
\hline Set monotonicity & $\begin{array}{l}A \subseteq B \Rightarrow R \downarrow A \subseteq R \downarrow B \\
A \subseteq B \Rightarrow R \uparrow A \subseteq R \uparrow B\end{array}$ & $\begin{array}{l}A \subseteq B \Rightarrow R \downarrow_{\mathcal{I}} A \subseteq R \downarrow_{\mathcal{I}} B \\
A \subseteq B \Rightarrow R \uparrow_{\mathcal{C}} A \subseteq R \uparrow_{\mathcal{C}} B\end{array}$ \\
\hline Relation monotonicity & $\begin{array}{l}R_{1} \subseteq R_{2} \Rightarrow R_{2} \downarrow A \subseteq R_{1} \downarrow A \\
R_{1} \subseteq R_{2} \Rightarrow R_{1} \uparrow A \subseteq R_{2} \uparrow A\end{array}$ & $\begin{array}{l}R_{1} \subseteq R_{2} \Rightarrow R_{2} \downarrow_{\mathcal{I}} A \subseteq R_{1} \downarrow_{\mathcal{I}} A \\
R_{1} \subseteq R_{2} \Rightarrow R_{1} \uparrow_{\mathcal{C}} A \subseteq R_{2} \uparrow_{\mathcal{C}} A\end{array}$ \\
\hline Intersection & $\begin{array}{l}R \downarrow(A \cap B)=R \downarrow A \cap R \downarrow B \\
R \uparrow(A \cap B) \subseteq R \uparrow A \cap R \uparrow B\end{array}$ & $\begin{array}{l}R \downarrow_{\mathcal{I}}(A \cap B)=R \downarrow_{\mathcal{I}} A \cap R \downarrow_{\mathcal{I}} B \\
R \uparrow_{\mathcal{C}}(A \cap B) \subseteq R \uparrow_{\mathcal{C}} A \cap R \uparrow_{\mathcal{C}} B\end{array}$ \\
\hline Union & $\begin{array}{l}R \downarrow(A \cup B) \supseteq R \downarrow A \cup R \downarrow B \\
R \uparrow(A \cup B)=R \uparrow A \cup R \uparrow B\end{array}$ & $\begin{array}{l}R \downarrow_{\mathcal{I}}(A \cup B) \supseteq R \downarrow_{\mathcal{I}} A \cup R \downarrow_{\mathcal{I}} B \\
R \uparrow_{\mathcal{C}}(A \cup B)=R \uparrow_{\mathcal{C}} A \cup R \uparrow_{\mathcal{C}} B \\
\end{array}$ \\
\hline Idempotence & $\begin{array}{l}R \downarrow(R \downarrow A)=R \downarrow A \\
R \uparrow(R \uparrow A)=R \uparrow A\end{array}$ & $\begin{array}{l}R \downarrow_{\mathcal{I}}\left(R \downarrow_{\mathcal{I}} A\right)=R \downarrow_{\mathcal{I}} A \\
R \uparrow_{\mathcal{C}}\left(R \uparrow_{\mathcal{C}} A\right)=R \uparrow_{\mathcal{C}} A \\
\end{array}$ \\
\hline Constant sets & $\begin{array}{l}R \downarrow \emptyset=\emptyset=R \uparrow \emptyset \\
R \downarrow U=U=R \uparrow U\end{array}$ & $\begin{array}{l}R \downarrow_{\downarrow_{\mathcal{I}}} \hat{\alpha}=\hat{\alpha} \\
R \uparrow_{\mathcal{C}} \hat{\alpha}=\hat{\alpha}\end{array}$ \\
\hline
\end{tabular}

Corollary 3. Let $\mathcal{T}$ and $\mathcal{S}$ be a t-norm and its $\mathcal{N}$-dual t-conorm. If $R$ is reflexive, and $(\mathcal{I}, \mathcal{C})=\left(\mathcal{I}_{\mathcal{S}, \mathcal{N}}, \mathcal{T}\right)$ or $(\mathcal{I}, \mathcal{C})=\left(\mathcal{I}_{\mathcal{T}}, \mathcal{T}\right)$, then the inclusion property holds.

Proposition 4. The properties of set and relation monotonicity, intersection and union always hold.

Proposition 5. If $R$ is a reflexive and $\mathcal{T}$-transitive relation, where $\mathcal{T}$ is a leftcontinuous t-norm and the pair $(\mathcal{I}, \mathcal{C})$ consists of the $R$-implicator $\mathcal{I}_{\mathcal{T}}$ and the t-norm $\mathcal{T}$, then the idempotence property holds.

Proposition 6. If $R$ is a reflexive relation, $\mathcal{I}$ a border implicator and $\mathcal{C}$ a border conjunctor, then the constant sets property holds.

Summing up, in order to satisfy all properties in Table $2, \mathcal{C}$ should be a leftcontinuous t-norm $\mathcal{T}$ and $\mathcal{I}$ its R-implicator, while $R$ needs to be at least reflexive and $\mathcal{T}$-transitive. Propositions 2 and 5 do not hold in general for S-implicators, for instance, Dubois and Prade's model [3] does not satisfy them.

\section{Axiomatic Approach}

In the axiomatic approach, we work with unary operators on $\mathcal{F}(U)$ and some axioms to obtain a fuzzy relation $R$ such that the operators behave as approxi- 
mation operators with respect to $R$. Such an approach is useful to get insight in the logical structure of fuzzy rough sets.

As our starting point, we use the axiomatic approach developed by $\mathrm{Wu}$ et al. [13], who propose axioms to characterise lower and upper approximations, which are generalized here for an implicator-conjunctor pair.

Definition 2. Let $H, L: \mathcal{F}(U) \rightarrow \mathcal{F}(U), \mathcal{C}$ a conjunctor and $\mathcal{I}$ an implicator. $H$ is a $\mathcal{C}$-upper approximation if it satisfies, for all $A, A_{j} \in \mathcal{F}(U), \alpha \in[0,1]$,

$$
\begin{aligned}
& \text { (H1) } H\left(\hat{\alpha} \cap_{\mathcal{C}} A\right)=\hat{\alpha} \cap_{\mathcal{C}} H(A) \\
& \text { (H2) } H\left(\bigcup_{j \in J} A_{j}\right)=\bigcup_{j \in J} H\left(A_{j}\right)
\end{aligned}
$$

$L$ is an $\mathcal{I}$-lower approximation if it satisfies, for all $A, A_{j} \in \mathcal{F}(U), \alpha \in[0,1]$,

$$
\begin{aligned}
& (L 1) L\left(\hat{\alpha} \Rightarrow_{\mathcal{I}} A\right)=\hat{\alpha} \Rightarrow_{\mathcal{I}} L(A) \\
& (L 2) L\left(\bigcap_{j \in J} A_{j}\right)=\bigcap_{j \in J} L\left(A_{j}\right)
\end{aligned}
$$

Wu et al. required $\mathcal{C}$ and $\mathcal{I}$ to be a continuous t-norm and implicator, resp., but these conditions can be slightly weakened. For this, we can use e.g. results from [22] obtained in the framework of fuzzy modal logics that can be easily adapted to approximation operators.

Proposition 7. Let $H: \mathcal{F}(U) \rightarrow \mathcal{F}(U)$ and $\mathcal{T}$ a left-continuous t-norm. $H$ is a $\mathcal{T}$-upper approximation if and only if for all $A \in \mathcal{F}(U), H(A)=R \uparrow_{\mathcal{T}} A$, where $R(x, y)=H(\{x\})(y)$, for $x, y$ in $U$.

Proposition 8. Let $L: \mathcal{F}(U) \rightarrow \mathcal{F}(U)$ and $\mathcal{I}$ an EP implicator that is leftcontinuous in its first argument and such that $\mathcal{N}_{\mathcal{I}}$ is continuous. $L$ is an $\mathcal{I}$ lower approximation if and only if for all $A \in \mathcal{F}(U), L(A)=R \downarrow_{\mathcal{I}} A$, where $R(x, y)=\mathcal{N}_{\mathcal{I}}(L(U \backslash\{x\})(y))$, for $x, y$ in $U$.

Adding more axioms to Definition 2, we can characterize specific properties of the fuzzy relation $R$, as the following propositions show.

Proposition 9. Let $\mathcal{T}$ be a left-continuous t-norm and $H$ a $\mathcal{T}$-upper approximation. There exists a fuzzy relation $R$ in $U$ such that $H=R \uparrow_{\mathcal{T}}$ that is

1. inverse serial $\Leftrightarrow \forall \alpha \in[0,1]: H(\hat{\alpha})=\hat{\alpha} \Leftrightarrow H(U)=U$

2. reflexive $\Leftrightarrow \forall A \in \mathcal{F}(U): A \subseteq H(A)$

3. symmetric $\Leftrightarrow \forall x, y \in U: H(\{x\})(y)=H(\{y\})(x)$

4. $\mathcal{T}$-transitive $\Leftrightarrow \forall A \in \mathcal{F}(U): H(H(A)) \subseteq H(A)$

Proposition 10. Let $\mathcal{I}$ be a border and EP implicator that is left-continuous in its first argument such that $\mathcal{N}_{\mathcal{I}}$ is continuous, and $L$ an $\mathcal{I}$-lower approximation. There exists a fuzzy relation $R$ in $U$ such that $L=R \downarrow_{\mathcal{I}}$ that is 
1. inverse serial $\Leftrightarrow \forall \alpha \in[0,1]: L(\hat{\alpha})=\hat{\alpha}$ and $\mathcal{I}$ satisfies $x \leq y \Leftrightarrow \forall z \in[0,1]$ : $\mathcal{I}(x, z) \geq \mathcal{I}(y, z)$

2. reflexive $\Leftrightarrow \forall A \in \mathcal{F}(U): L(A) \subseteq A$

3. symmetric $\Leftrightarrow \forall x, y \in U, \alpha \in[0,1]: L\left(\{x\} \Rightarrow_{\mathcal{I}} \hat{\alpha}\right)(y)=L\left(\{y\} \Rightarrow_{\mathcal{I}} \hat{\alpha}\right)(x)$

4. $\mathcal{T}$-transitive $\Leftrightarrow \forall A \in \mathcal{F}(U): L(L(A)) \subseteq L(A)$ for all $A$ in $\mathcal{F}(U)$ and $\mathcal{I}$ satisfies $\mathcal{I}(x, \mathcal{I}(y, z))=\mathcal{I}(\mathcal{T}(x, y), z)$ for all $x, y, z$ in $[0,1]$

The above propositions characterize lower and upper approximations separately. If these operators are dual, we can link them together.

Proposition 11. Let $\mathcal{T}$ be a left-continuous t-norm, $\mathcal{I}$ an EP implicator that is left-continuous in its first argument and such that $\mathcal{N}_{\mathcal{I}}$ is involutive, $H$ a $\mathcal{T}$-upper approximation and $L$ an $\mathcal{I}$-lower approximation. If $H$ and $L$ satisfy duality w.r.t. $\mathcal{N}_{\mathcal{I}}$, then there exists a binary fuzzy relation $R$ in $U$ such that $H=R \uparrow_{\mathcal{I}}$ and $L=R \downarrow_{\mathcal{I}}$.

A drawback of the above approach is that it excludes some important operators. For instance, it can be verified that the R-implicator $\mathcal{I}_{\text {min }}$ does not satisfy the conditions of Proposition 8 , because $\mathcal{N}_{\mathcal{I}_{\min }}$ is not involutive. However, it satisfies all properties from Table 2. For this reason, below we introduce and characterize the alternative notion of a $\mathcal{T}$-coupled pair of approximations.

Definition 3. Let $\mathcal{T}$ be a left-continuous t-norm, $H, L: \mathcal{F}(U) \rightarrow \mathcal{F}(U)$. We call $(H, L)$ a $\mathcal{T}$-coupled pair of upper and lower approximations if the following conditions hold:

(H1,H2) $H$ is a $\mathcal{T}$-upper fuzzy approximation operator

(L2) $L\left(\bigcap_{j \in J} A_{j}\right)=\bigcap_{j \in J} L\left(A_{j}\right)$

(HL) $L\left(A \Rightarrow_{\mathcal{I}_{\mathcal{T}}} \hat{\alpha}\right)=H(A) \Rightarrow_{\mathcal{I}_{\mathcal{T}}} \hat{\alpha}$

Proposition 12. Let $\mathcal{T}$ be a left-continuous t-norm, $H, L: \mathcal{F}(U) \rightarrow \mathcal{F}(U)$. $(H, L)$ is a $\mathcal{T}$-coupled pair of upper and lower approximations if and only if there exists a binary fuzzy relation $R$ in $U$ such that $H=R \uparrow_{\mathcal{T}}$ and $L=R \downarrow_{\mathcal{I}_{\mathcal{T}}}$.

Proof. Assume $(H, L)$ is a $\mathcal{T}$-coupled pair and $A \in \mathcal{F}(U)$. By (H1, H2), $H$ is a $\mathcal{T}$-upper approximation, so by Proposition $7, H(A)=R \uparrow_{\mathcal{T}} A$, where $R(x, y)=$ $H(\{x\})(y)$, for $x, y$ in $U$. On the other hand, it can be verified that $A=$ $\bigcap_{y \in U}\left(\{y\} \Rightarrow_{\mathcal{I}_{\mathcal{T}}} \widehat{A(y)}\right)$, so by (L2) and (HL), we have $L(A)=\bigcap_{y \in U} L\left(\{y\} \Rightarrow_{\mathcal{I}_{\mathcal{T}}}\right.$ $\widehat{A(y)})=\bigcap_{y \in U} H(\{y\}) \Rightarrow_{\mathcal{I}_{\mathcal{T}}} \widehat{A(y)}=R \downarrow_{\mathcal{I}_{\mathcal{T}}} A$.

Conversely, it is clear that $R \uparrow_{\mathcal{I}}$ and $R \downarrow_{\mathcal{I}_{\mathcal{T}}}$ are an upper and a lower approximation satisfying (H1, H2) and (L2), respectively. To see (HL), let $x \in U, \alpha \in[0,1]$, then $\left(R \downarrow_{\mathcal{I}_{\mathcal{T}}}\left(A \Rightarrow_{\mathcal{I}_{\mathcal{T}}} \hat{\alpha}\right)\right)(x)=\inf _{y \in U} \mathcal{I}_{\mathcal{T}}\left(R(y, x), \mathcal{I}_{\mathcal{T}}(A(y), \alpha)\right)=\inf _{y \in U} \mathcal{I}_{\mathcal{T}}(\mathcal{T}(R(y, x)$, $A(y)), \alpha)=\mathcal{I}_{\mathcal{T}}\left(\sup _{y \in U} \mathcal{T}(R(y, x), A(y)), \alpha\right)=\mathcal{I}_{\mathcal{T}}\left(\left(R \uparrow_{\mathcal{T}} A\right)(x), \alpha\right)=\left(R \uparrow_{\mathcal{T}} A \Rightarrow_{\mathcal{I}_{\mathcal{T}}}\right.$ $\hat{\alpha})(x)$. 
Proposition 13. Let $\mathcal{T}$ be a left-continuous t-norm and let $(H, L)$ be a $\mathcal{T}$ coupled pair of upper and lower fuzzy approximation operators. There exists a binary fuzzy relation $R$ in $U \times U$ such that $H=R \uparrow_{\mathcal{T}}$ and $L=R \downarrow_{\mathcal{I}_{\mathcal{T}}}$ that is:

1. inverse serial $\Leftrightarrow H(U)=U \Leftrightarrow \forall A \in \mathcal{F}(U): L(A) \subseteq H(A)$

2. reflexive $\Leftrightarrow \forall A \in \mathcal{F}(U): L(A) \subseteq A \Leftrightarrow \forall A \in \mathcal{F}(U): A \subseteq H(A)$

3. symmetric $\Leftrightarrow \forall x, y \in U: H(\{x\})(y)=H(\{y\})(x) \Leftrightarrow \forall A \in \mathcal{F}(U): H(L(A)) \subseteq$ $A \Leftrightarrow \forall A \in \mathcal{F}(U): A \subseteq L(H(A))$

4. $\mathcal{T}$-transitive $\Leftrightarrow \forall A \in \mathcal{F}(U): L(A) \subseteq L(L(A)) \Leftrightarrow \forall A \in \mathcal{F}(U): H(H(A)) \subseteq$ $H(A)$

Proof. By Proposition 12, we know that there exists a relation $R$ such that $H=R \uparrow_{\mathcal{T}}$ and $L=R \downarrow_{\mathcal{I}_{\mathcal{T}}}$.

1. The equivalence between inverse seriality and $H(U)=U$ can be proved as follows: $H(U)(x)=\sup _{y \in U} \mathcal{T}(R(y, x), U(y))=\sup _{y \in U} \mathcal{T}(R(y, x), 1)=$ $\sup _{y \in U} R(y, x)$. Hence, $U=H(U)$ iff $H(U)(x)=1$ for all $x \in U$, iff $\sup _{y \in U} R(y, x)=1$ for all $x \in U$. The equivalence with $L(A) \subseteq H(A)$ for all $A \in \mathcal{F}(U)$ corresponds to [22, Proposition 4].

2. This corresponds to [22, Proposition 5].

3. The first equivalence is proved as in Proposition 9, item 3. The second and third one correspond to [22, Proposition 9].

4. This corresponds to [22, Proposition 13].

\section{Conclusion and Future Work}

In this paper, we have studied a general implicator-conjunctor based model for the lower and upper approximation of a fuzzy set under a binary fuzzy relation. We reviewed models from the literature that can be seen as special cases, and enriched the existing axiomatic approach with a new notion of $\mathcal{T}$-coupled pairs of approximations, which characterize the operations satisfying all relevant properties of classical rough sets, i.e., left-continuous t-norms and their R-implicators.

An important challenge is to extend the formal treatment to noise-tolerant fuzzy rough set models, such as those studied in [23-29]. Observing that the implicator-conjunctor based approximations are sensitive to small changes in the arguments (for instance, because of their reliance on inf and sup operations), many authors have proposed models that are more robust against data perturbation. However, this normally goes at the expense of the properties the corresponding fuzzy rough set model satisfies.

\section{Acknowledgment}

This work was partially supported by the Spanish Ministry of Science and Technology under Project TIN2011-28488. Lluis Godo has been partially supported by the MINECO Project TIN2012-39348-C02-01. 


\section{References}

1. Zadeh, L.A.: Fuzzy sets. Information and control 8 (1965) 338-353

2. Pawlak, Z.: Rough sets. International journal of computer and information sciences 11(5) (1982) 341-356

3. Dubois, D., Prade, H.: Rough fuzzy sets and fuzzy rough sets. International Journal of General Systems 17 (1990) 191-209

4. Jensen, R., Shen, Q.: Fuzzy-rough sets assisted attribute selection. IEEE Transactions on Fuzzy Systems 15(1) (2007) 73-89

5. Tsang, E., Chen, D., Yeung, D., Wang, X., Lee, J.: Attributes reduction using fuzzy rough sets. IEEE Transactions on Fuzzy Systems 16(5) (2008) 1130-1141

6. Cornelis, C., Hurtado Martín, G., Jensen, R., Ślȩzak, D.: Attribute selection with fuzzy decision reducts. Information Sciences 180(2) (2010) 209-224

7. Verbiest, N., Cornelis, C., Herrera, F.: FRPS: A fuzzy rough prototype selection method. Pattern Recognition 46(10) (2013) 2770-2782

8. Morsi, N., Yakout, M.: Axiomatics for fuzzy rough set. Fuzzy Sets Systems 100 (1998) 327-342

9. Radzikowska, A., Kerre, E.: A comparative study of fuzzy rough sets. Fuzzy Sets and Systems 126 (2002) 137-155

10. Wu, W., Mi, J., Zhang, W.: Generalized fuzzy rough sets. Information Sciences 151 (2003) 263-282

11. Mi, J., Zhang, W.: An axiomatic characterization of a fuzzy generalization of rough sets. Information Sciences 160 (2004) 235-249

12. Wu, W., Zhang, W.: Constructive and axiomatic approaches of fuzzy approximation operators. Information Sciences 159 (2004) 233-254

13. Wu, W., Leung, Y., Mi, J.: On characterizations of (I, T)-fuzzy rough approximation operators. Fuzzy Sets and Systems 154 (2005) 76-102

14. Pei, D.: A generalized model of fuzzy rough sets. International Journal of General Systems 34(5) (2005) 603-613

15. Yeung, D., Chen, D., Tsang, E., Lee, J., Xizhao, W.: On the generalization of fuzzy rough sets. IEEE Transactions on Fuzzy Systems 13(3) (2005) 343-361

16. De Cock, M., Cornelis, C., Kerre, E.: Fuzzy rough sets: the forgotten step. IEEE Transactions on Fuzzy Systems 15(1) (2007) 121-130

17. Mi, J., Leung, Y., Zhao, H., Feng, T.: Generalized fuzzy rough sets determined by a triangular norm. Information Sciences 178 (2008) 3203-3213

18. Hu, Q., Zhang, L., Chen, D., Pedrycz, W., Yu, D.: Gaussian kernel based fuzzy rough sets: model, uncertainty measures and applications. International Journal of Approximate Reasoning 51 (2010) 453-471

19. Slowinski, R., Vanderpooten, D.: Similarity relations as a basis for rough approximations. In Wang, P., ed.: Advances in Machine Intelligence and Soft Computing. (1997) 17-33

20. Slowinski, R., Vanderpooten, D.: Generalized rough set models. In L. Polkowski, A.S., ed.: Rough sets in knowledge discovery. (1998) 286-318

21. Fodor, J.: Left-continuous t-norms in fuzzy logic: an overview. Journal of applied sciences at Budapest Tech Hungary 1(2) (2004)

22. Radzikowska, A., Kerre, E.: Characterisation of main classes of fuzzy relations using fuzzy modal operators. Fuzzy Sets and Systems 152 (2005) 223-247

23. Salido, J.M.F., Murakami, S.: Rough set analysis of a general type of fuzzy data using transitive aggregations of fuzzy similarity relations. Fuzzy Sets and Systems 139 (2003) 635-660 
24. Mieszkowicz-Rolka, A., Rolka, L.: Fuzzy rough approximations of process data. International Journal of Approximate Reasoning 49 (2008) 301-315

25. Cornelis, C., Cock, M.D., Radzikowska, A.: Vaguely quantified rough sets. In: Proceedings of 11th International Conference on Rough Sets, Fuzzy Sets, Data Mining and Granular Computing (RSFDGrC2007). (2007) 87-94

26. Zhao, S., Tsang, E.C.C., Chen, D.: The model of fuzzy variable precision rough sets. IEEE Transactions on Fuzzy Systems 17(2) (2009) 451-467

27. Hu, Q., An, S., Yu, D.: Soft fuzzy rough sets for robust feature evaluation and selection. Information Sciences 180 (2010) 4384-4400

28. Cornelis, C., Verbiest, N., Jensen, R.: Ordered weighted average based fuzzy rough sets. In: Proceedings of the 5th International Conference on Rough Sets and Knowledge Technology (RSKT2010). (2010) 78-85

29. Hu, Q., Zhang, L., An, S., Zhang, D., Yu, D.: On robust fuzzy rough set models. IEEE Transactions on Fuzzy Systems 20(4) (2012) 636 - 651 